OPEN ACCESS

Edited by:

Zongbing You,

Tulane University, United States

Reviewed by:

Daotai Nie,

Southern Illinois University School of Medicine, United States

Benyi Li,

University of Kansas Medical Center

United States

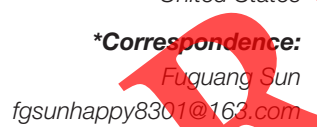

Specialty section:

This article was submitted to

Surgical Oncology,

a section of the journa

Frontiers in Oncology

Received: 27 December 2019

Accepted: 09 June 2020

Published: 06 August 2020

Citation:

Zhao Z, Liang S and Sun F (2020)

LncRNA DLX6-AS1 Promotes

Malignant Phenotype and Lymph

Node Metastasis in Prostate Cancer

by Inducing LARGE Methylation.

Front. Oncol. 10:1172.

doi: 10.3389/fonc.2020.01172

\section{LncRNA DLX6-AS1 Promotes Malignant Phenotype and Lymph Node Metastasis in Prostate Cancer by Inducing LARGE Methylation}

\author{
Zhifeng Zhao ${ }^{1}$, Shuxia Liang ${ }^{2}$ and Fuguang Sun ${ }^{1 *}$ \\ ${ }^{1}$ Department of Urology, Linyi People's Hospital of Shandong Province, Linyi, China \\ Hospital of Shandong Province, Linyi, China
}

Long non-coding RNAs (IncRNAs) have recently become recognized as crucial players in cancer cellular events including proliferation, migration, and invasion. Herein, we investigated the potential role of IncRNA DLX6-AS1 in prostate cancer cell malignant behaviors and lymph node metastasis. A differentially expressed IncRNA DLX6-AS1 and its downstream regulatory gene (LARGE) were predicted by analysis in silico. RT-qPCR and western blot analysis results demonstrated that DLX6-AS1 was highly expressed, but LARGE was poorly expressed in prostate cancer tissues and cells. The online website indicated that DLX6-AS1 negatively targeted LARGE expression, which was validated by Pearson conelation analysis and MSP. ChIP, RIP, and RNA pull-down assays further suggested that DLX6-AS1 downregulated LARGE expression through recruitment of DNMT1 to its promater. Weinduced DLX6-AS1/LARGE overexpression or knockdown to examine their effects through Edu and Transwell assays, which revealed that DLX6-AS1 overexpression accelerated proliferation, invasion, and migration of prostate cancer cells, and that overexpression of LARGE rescued these effects. Tumors xenografts studies confirmed that DLX6-AS1 promoted lymph node metastasis by regulating LARGE, as evidenced by enhanced expression of MMP-9, UPAR, and cathepsin B. In summary, DLX6-AS1 stimulated prostate cancer malignant progression and lymph node metastasis by inducing DNMT1-mediated LARGE methylation, highlighting a potential therapeutic target against prostate cancer.

Keywords: prostate cancer, LncRNA homeobox 6 antisense 1, DNA methyltransferase 1, like-acetylglucosaminyltransferase, $\mathrm{CpG}$ island methylation

\section{INTRODUCTION}

Prostate cancer is the most prevalent malignancy in the male population, ranking in second place as a cause of male cancer-associated death (1). Of note, lymph node status is a vital prognostic indicator of outcome for prostate cancer (2). In the past 10 years, great progresses have been made with the advent of new hormonal agents and chemotherapy regimens combined with standard androgen-deprivation therapy for prostate cancer. However, the application of systemic therapy alone may lead to a castrate-resistant disease state. Additional survival benefits could potentially be achieved with local cytoreductive and/or metastasis-directed therapies (3). 
Long non-coding RNAs (lncRNAs) are defined as RNA transcripts of length exceeding 200 nucleotides, which do not express proteins, but exert regulatory effects on gene transcription (4). In clinical practice, IncRNAs are emerging as important tools for molecular research and for the detection of new biomarkers, as well as serving as promising therapeutic targets for genitourinary oncology (5). For instance, lncRNA distal-less homeobox 6 antisense 1 (DLX6-AS1) was found to be an oncogene in renal cell carcinoma, and enhanced expression of DLX6-AS1 was positively linked to tumor progression and development (6). On the other hand, exogenous expression of like-acetylglucosaminyltransferase (LARGE) in cancer cells accelerated the glycosylation and laminin binding of $\alpha$-dystroglycan, culminating in restored cell adhesion and diminished cell migration in vitro (7). Furthermore, DNA methyltransferase 1 (DNMT1) has extensive expression in gastric (8), colon (9), lung (10), laryngeal (11) cancer cells; and downregulated DLX6-AS1 re-differentiates liver cancer stem cells through promotion of CADM1 by suppressing the methylation of the CADM1 promoter (12). However, the targeting relationship between DLX6-AS1 and LARGE has not yet been reported. Based on our preliminary predictions and some previous studies, we hypothesized that DLX6-AS1 contributes to stimulating the progression of prostate cancer via effects on the regulation of LARGE. Thus, we tested the potential function of DLX6-AS1 in prostate cancer cell proliferation, invasion, and lymph node metastasis, aiming to substantiate a new therapeutic target for the treatment of prostate cancer.

\section{METHODS}

\section{Ethics Statement}

The study was conducted under the approval of the Ethics Committee of Linyi People's Hospital of Shandong Province. All participants or their guardians signed informed consent. The animal study was reviensed and approved by Linyi People's Hospital of Shandong Province and the experiments involving animals were performed in compliance with the recommendations in the Guide for the Care and Use of Laboratory Animals of the National Institutes of Health.

\section{Bioinformatic Analysis}

Prostate cancer dataset GSE55945 and its probe annotation files were downloaded from the Gene Expression Omnibus database. The "limma" package of $\mathrm{R}$ language software was utilized for differential analysis of gene expression, with |log FoldChange| $>1, p<0.05$ as the screening criteria, with depiction of differentially expressed genes as box-plots.

\footnotetext{
Abbreviations: ANOVA, analysis of variance; ChIP, chromatin immunoprecipitation; DLX6-AS1, distal-less homeobox 6 antisense 1; DNMT1, DNA methyltransferase 1; FBS, fetal bovine serum; FISH, fluorescence in situ hybridization; GAPDH, glyceraldehyde-3-phosphate dehydrogenase; HE, hematoxylin-eosin; IncRNAs, long non-coding RNAs; LARGE, likeacetylglucosaminyltransferase; MSP, methylation-specific PCR; PBS, phosphate buffer solution; PSA, prostate specific antigen; RPMI 1640, Roswell Park Memorial Institute 1640; shRNAs, short hairpin RNAs; RT-qPCR, reverse transcription quantitative polymerase chain reaction.
}

\section{Sample Collection}

From March 2016 to September 2017, patients with prostate cancer ( $n=32$, aged $49.5 \pm 6.2$ years) and benign prostatic hyperplasia $(n=28$, aged $48.61 \pm 4.98$ years $)$ admitted in Linyi People's Hospital of Shandong Province were recruited for this study. Surgical or biopsy specimens of their cancerous tissues (with at least $80 \%$ tumor cells confirmed by pathological examination) or benign prostatic hyperplasia tissues were collected. The patients with prostate cancer were diagnosed according to the World Health Organization criteria. No patient had received any anticancer treatment prior to the operation. All tumor nodules confirmed by pathological examination were completely resected. Complete clinicopathological and follow-up data were obtained. Patients who died from non-prostate cancer or accidents were excluded. Amongst the 32 cancer patients, 4 patients had PSA level $<4.0 \mathrm{ng} / \mathrm{mL}, 17$ had PSA level ranging from 4.0 to $10.0 \mathrm{ng} / \mathrm{mL}$, and 11 had PSA level $>10.0 \mathrm{ng} / \mathrm{mL}$. In patients with benign prostatic hyperplasia, 5 had PSA level $<4.0 \mathrm{ng} / \mathrm{mL}, 13 \mathrm{had}$ PSA level between 4.0 and $10.0 \mathrm{ng} / \mathrm{mL}$, and 10 had PSA level $>10.0 \mathrm{ng} / \mathrm{nL}$. Meanwhile, 7 of 32 prostate cancer tissues were of volume $<30 \mathrm{~mL}$, 4 were $30-50 \mathrm{~mL}$ and 21 were $>50 \mathrm{~mL}$. For benign prostatic hyperplasia, 5 samples were of volume $<30 \mathrm{~mL}$, 6 were $30-50 \mathrm{~mL}$, and 17 exceeded $50 \mathrm{~mL}$.

\section{Cell Culture}

The prostate cancer cell lines CWR22rv1, LAPC-9, DU145, LNCaP, and PC-3M, and normal human prostate epithelial cell line PrEC were purchased from the Cell Bank of the Chinese Academy of Sciences (Shanghai, China; http://www.cellbank. org.cn/index.asp). The cells were cultured in Roswell Park Memorial Institute 1640 (RPMI 1640) medium (Life Technology, Carlsbad, CA, USA) supplemented with $10 \%$ fetal bovine serum (FBS) and incubated at $37^{\circ} \mathrm{C}$ with $5 \% \mathrm{CO}_{2}$. Upon reaching $50 \%$ confluence, cells were treated with the methyltransferase inhibitor 5-aza-deoxycytidine (Aza-dC; $10 \mu \mathrm{M}$, Sigma-Aldrich Chemical Company, St Louis, MO, USA), cultured for 4 days, and then total RNA was extracted for RNA analysis.

\section{Cell Transduction}

pLV-EGFP-N was applied to construct overexpression, including overexpressed (oe)-DLX6-AS1 and oe-LARGE. pSIH1-H1copGFP was applied to construct short hairpin RNAs (shRNAs) of DLX6-AS1 (sh-DLX6-AS1), DNMT1 (sh-DNMT1), and LARGE (sh-LARGE) and negative control (sh-NC) (all from GenePharma Co., Ltd., Shanghai, China). Prostate cancer cells in logarithmic growth phase were trypsinized to prepare cell suspensions at the cell density of $5 \times 10^{4}$ cells $/ \mathrm{mL}$, and then seeded on a 6-well plate ( $2 \mathrm{~mL} /$ well). After incubation overnight at $37^{\circ} \mathrm{C}$, the cells were infected with lentivirus $\left(1 \times 10^{8}\right.$ lentivirus $/ \mathrm{mL}$ ). Cells were harvested $48 \mathrm{~h}$ post-transduction to determine the efficiency of infection.

\section{RT-qPCR}

Total mRNA was isolated using the Trizol kit (Invitrogen Inc., Carlsbad, CA, USA). RNA quality and concentration were assessed by ultraviolet spectrophotometry (ND-1000, NanoDrop Technologies, Wilmington, DE, USA). A PrimeScript RT Reagent 
TABLE 1 | The primer sequences for RT-qPCR.

\begin{tabular}{|c|c|c|}
\hline Target gene & Sequences $\left(5^{\prime}-3^{\prime}\right)$ & Target size (bp) \\
\hline \multirow[t]{2}{*}{ DLX6-AS1 } & F: 5'-TCAGGCCTGGTCAACAGGTA-3' & 165 \\
\hline & R: 5'-CAGGTCTCGTCCACCCAATC-3' & \\
\hline \multirow[t]{2}{*}{ LARGE } & F: 5'-CTGCCTTGTGTCTGGAATGTGC-3' & 106 \\
\hline & R: 5'-GCTTCTTTGGTGAGTTCCAGTGG-3' & \\
\hline \multirow[t]{2}{*}{ DNMT1 } & F: 5'-GAAGGAGCCCGTGGATG-3' & 241 \\
\hline & R: 5'-GTTGATGTCTGCGTGGTAG-3' & \\
\hline \multirow[t]{2}{*}{ DNMT3a } & F: 5'-TACGCACCACCTCCAC-3' & 139 \\
\hline & R: 5'-AGATGTCCTCAATGTTCC-3' & \\
\hline \multirow[t]{2}{*}{ DNMT3b } & F: 5'-CGACCTCACAGACGACAC-3' & 170 \\
\hline & R: 5'-TTCCAAАСТССТTCCСАТСC-3' & \\
\hline \multirow[t]{2}{*}{ MMP-9 } & F: 5'-GTGGCACCACCACAACAT-3' & 495 \\
\hline & R: 5'-TCCTGGGTGTAGAGTCTCTCG-3' & \\
\hline \multirow[t]{2}{*}{ UPAR } & F: 5'-TAAGACCAACGGGGATTGCC-3' & 194 \\
\hline & R: 5'-ACACAACCTCGGTAAGGCTG-3' & \\
\hline \multirow[t]{2}{*}{ Cathepsin B } & F: 5'-GCTTCGATGCACGGGAACAATG-3' & 132 \\
\hline & R: 5'-CATTGGTGTGGATGCAGATCCG-3' & \\
\hline \multirow[t]{2}{*}{ GAPDH } & F: 5'-GGTATCGTGGAAGGACTCATGAC-3' & 188 \\
\hline & R: 5'-ATGCCAGTGAGCTTCCCGTTCAG-3' & \\
\hline
\end{tabular}

$F$, forward; $R$, reverse.

Kit (Takara Biotechnology Ltd., Dalian, Liaoning, China) was used for the reverse transcription of total RNA (400 ng). Realtime PCR was performed by the SYBR ${ }^{\circledR}$ Premix Ex Taq ${ }^{\mathrm{TM}}$ II (Tli RNaseH Plus) kit (Takara Holdings Inc., Kyoto, Japan) on a Thermal Cycler Dice Real Time System Amplifier (TP800, Takara Holdings Inc., Kyoto, Japan). The primers were synthesized by Guangzhou RiboBio Co., Ltd. (Guangzhou, Guangdong, China) (Table 1). Relative gene expression was calculated using the $2^{-\Delta \Delta C t}$ method and normalized to glyceraldehyde-3-phosphate dehydrogenase (GAPDH).

\section{Western Blot Analysis}

Total proteins were extracted using a cell lysis buffer (C0481, Sigma-Aldrich Chemical Company) at $4^{\circ} \mathrm{O}$ for $30 \mathrm{~min}$, subjected to sodium dodecyl sulfate-polyacrylamide gel electrophoresis and then transferred onto the polyvinylidene fluoride membrane (Millipore, Billerica, MA, USA). After being sealed with 5\% skim milk for $1 \mathrm{~h}$, the membrane was incubated overnight at $4^{\circ} \mathrm{C}$ with rabbit antiboenes (diluted with Tris-buffered saline Tween-20; Abcam Inc., Cambridge, UK) to LARGE (ab228953, 1:200), DNMT1 (ab19905, 1:100), DNMT3b (ab79822, 1:1,000), DNMT3a (ab4897, 1:1,000), matrix metalloproteinase 9 (MMP9; ab73734, 1:100), Urokinase plasminogen activator receptor (uPAR; ab8222, 1:200), cathepsin B (ab125067, 1:1,000), and GAPDH (ab37168, 1:100). Afterwards, the membrane was further incubated with the secondary horseradish peroxidaseconjugated mouse anti-rabbit antibody (ab9482, 1:5,000; Abcam Inc.) at room temperature for $1 \mathrm{~h}$. Finally, the protein bands were visualized using an enhanced chemiluminescence detection system (Baomanbio, Shanghai, China) and quantified by Image J software. The relative protein expression was normalized to endogenous GAPDH.
TABLE 2 | The primer sequences for RT-qPCR in subcellular fractionation.

\begin{tabular}{llc}
\hline Target gene & Sequences $\left(\mathbf{5}^{\prime} \mathbf{- 3} \mathbf{3}^{\prime}\right)$ & Target size (bp) \\
\hline U6 & F: 5'-CTCGCTTCGGCAGCACATATAC-3' & 94 \\
& R: 5'-AACGCTTCACGAATTGCGTGTC-3' & \\
12S rRNA & F: 5'-TAGAGGAGCCTGTTCTGTAATCGA-3' & 111 \\
& R: 5'-TGCGCTTACTTTTAGCCTTCAT-3' & \\
45S rRNA & F: 5'-GAACGGTGGTGTGTCGTT-3' & 130 \\
& R: 5'-GCGTCTCGTCTCGTCTCACT-3' & \\
\hline
\end{tabular}

$F$, forward; $R$, reverse.

\section{Fluorescence in situ Hybridization (FISH)}

The cell slides were seeded into a 24 -well-culture plate at $5 \times$ $10^{3}$ cells/well for a 24 -h incubation. The cells were fixed by $4 \%$ paraformaldehyde at room temperature for $10 \mathrm{~min}$, and treated with $0.5 \%$ Triton-X in phosphate buffered saline (PBS) followed by pre-hybridization at $37^{\circ} \mathrm{C}$. The cells were then hybridized with hybridization solution containing DLX6-AS1 probes, stained with DAPI and mounted in dark. Finally, the specimens were observed under a laser confocal microscope (ECLIPSE E800; Nikon Instruments Inc., Melville, NY, USA).

\section{Subcellular Fractionation Assay}

The cells were separated by a PARIS ${ }^{\mathrm{TM}}$ Kit Protein and RNA Isolation System (Life Technology, Carlsbad, CA, USA) kit. In brief, cells were resuspended in $500 \mu \mathrm{L}$ cell fractionation buffer and placed on ice for 5-10 min after trypsinization and centrifugation. Then, the cells were centrifuged at $4^{\circ} \mathrm{C}$ at $500 \times \mathrm{g}$ for 5 min. The supernatant (cytoplasmic fraction) was transferred to a fresh $2 \mathrm{~mL}$ sterile and enzyme-free tube and centrifuged at $4^{\circ} \mathrm{C}$ at $500 \times \mathrm{g}$ for $5 \mathrm{~min}$. The precipitation (nuclear fraction) was lysed with $500 \mu \mathrm{L}$ cell fractionation buffer and $500 \mu \mathrm{L}$ cell disruption buffer. The cytoplasmic and nuclear fraction were separately washed with $500 \mu \mathrm{L} 2 \times$ Lysis/binding solution. The fractions were mixed with $500 \mu \mathrm{L}$ absolute ethanol and transferred into a filter cartridge. RNA from different fractions was harvested after washed in $500 \mu \mathrm{L}$ wash solution and eluted in $40 \mu \mathrm{L}$ elution solution for $30 \mathrm{~s}$. RT-qPCR was applied to assess the expression of lncRNA DLX6-AS1. 12S rRNA and GAPDH were used as positive controls of cytoplasmic RNA expression, and U6 and 45S rRNA were used as positive controls of nuclear RNA expression. The sequences of primers are shown in Table 2.

\section{Methylation-Specific PCR (MSP)}

Genomic DNA was extracted from cells using a genomic DNA extraction kit (TIANGEN Biotechnology Co., Ltd., Beijing, China). The concentration of purified total DNA was determined and optical density (OD) was measured at 260 and $280 \mathrm{~nm}$ according to the manual. DNA was modified with bisulfite by an EZ DNA methylation kit (TIANGEN Biotechnology Co., Ltd.). Bisulfite converted unmethylated cytosine to thymine, while methylated cytosine remained intact. Promoter methylation of LARGE was determined by MSP. A pair of primers were applied to assess the methylation-free sequence of $\mathrm{CpG}$ sites (modified by bisulfite), and the other pair was applied to amplify the 
TABLE 3 | The MSP with primers for methylated (M-MSP) and unmethylated (U-MSP) DNA.

\begin{tabular}{|c|c|}
\hline DNA & Sequences $\left(5^{\prime}-3^{\prime}\right)$ \\
\hline \multirow[t]{2}{*}{ LARGE-M-MSP } & F: 5'-TाITGAGTAGTTGGGATTATAGGC-3' \\
\hline & R: 5'-ACTITAAAAAACTAAAACGAACGAA-3' \\
\hline \multirow[t]{2}{*}{ LARGE-U-MSP } & F: 5'-TITGAGTAGTTGGGATTATAGGTGT-3' \\
\hline & R: 5' -ACTITAAAAAACTAAAACAAACAAA-3' \\
\hline
\end{tabular}

methylation-specific sequence of $\mathrm{CpG}$ loci (without bisulfite treatment) (Table 3). The total genome of methylated Hela cells (Takara Biotechnology Ltd., Dalian, Liaoning, China) was used as a positive control with water as NC. The total volume of the amplification reaction system was $25 \mu \mathrm{L}$, including $12.5 \mu \mathrm{L}$ Hot-StarTaq Master Mix (Qiagen company, Hilden, Germany), $1 \mu \mathrm{L}$ DNA template treated with bisulfite and $1.5 \mu \mathrm{L}$ forward and reverse primers, respectively. The product was separated by electrophoresis at $120 \mathrm{~V}$ with $1.5 \%$ agarose gel, stained with ethidium bromide and processed by image processing in the gel imaging system.

\section{RNA Immunoprecipitation (RIP) Assay}

A Magna RIP ${ }^{\mathrm{TM}}$ RNA-Binding Protein Immunoprecipitation Kit (Millipore, Billerica, MA, USA) was utilized in this experiment. RIP lysis buffer was applied to lysed prostate cancer cells upon reaching $90 \%$ confluence. Cell extracts $(100 \mu \mathrm{L})$ were coimmunoprecipitated with $900 \mu \mathrm{L}$ RIP buffer containing DNMT1 rabbit antibody (ab4897, 1:1,000, Abcam Inc.), and the retrieved RNA was subjected to RT-qPCR analysis. Normal mouse IgG was used as NC. Following centrifugation, 500 $\mu \mathrm{L}$ of RIP Wash Buffer was added into the centrifuge tube. After the supernatant was discarded, 50 $\mu \mathrm{L}$ RIP Wash Buffer was added with the loading buffer. Western blot analysis was adopted to detect whether the magnetic beads were coated with antibodies. The sample was incubated with proteinase $\mathrm{K}$ at $55^{\circ} \mathrm{C}$ for $30 \mathrm{~min}$ in a centrifuge tube After the protein was digested, immunoprecipitated RNA was separated and RT-qPCR was performed to detect the enrichment of DLX6-AS1.

\section{RNA Pull-Down Assay}

DLX6-AS1 RNA fragments were synthesized by T7 RNA polymerase (Ambion, Company, Austin, TX, USA) in vitro, treated with RNeasy Plus Mini kit and DNase I (Qiagen company, Hilden, Germany), then purified by RNeasy Mini Kit. The purified RNA $3^{\prime}$ end was labeled with biotin RNA labeling mixture (Ambion, Company, Austin, TX, USA). A total of 1 $\mu \mathrm{g}$ labeled RNA was heated in RNA structure buffer containing $10 \mathrm{mM}$ Tris $(\mathrm{pH}=7), 0.1 \mathrm{mM} \mathrm{KCl}, 10 \mathrm{mM} \mathrm{MgCl}_{2}$ at $95^{\circ} \mathrm{C}$ for $2 \mathrm{~min}$, incubated on ice for $3 \mathrm{~min}$, and then allowed to stand at room temperature for $30 \mathrm{~min}$ to form a suitable secondary structure of RNA. Prostate cancer cells $(3 \mu \mathrm{g})$ were lysed with cell lysis buffer (Sigma-Aldrich Chemical Company) at $4^{\circ} \mathrm{C}$ for $1 \mathrm{~h}$. The lysate was centrifuged at $12,000 \times \mathrm{g}$ at $4^{\circ} \mathrm{C}$ for $10 \mathrm{~min}$. Then, the supernatant was collected and transferred to RNasefree centrifugal tube. The biotinylated RNA (400 ng) was added
TABLE 4 | The primer sequences of LARGE promoter.

\begin{tabular}{lll}
\hline Target & Sequences $\left(\mathbf{5}^{\prime} \mathbf{- 3} \mathbf{3}\right)$ & Target size (bp) \\
\hline LARGE & F: 5'-AGGGTCCTGGAGAGGGAATCA-3' $^{\prime} 214$ \\
& R: 5' $^{\prime}$-TCTCTGGGAGATGGGGAAGG-3' & \\
\hline
\end{tabular}

with $500 \mu \mathrm{L}$ RIP buffer and incubated at room temperature for $1 \mathrm{~h}$ with the cell lysate. At the same time, part of the cell lysate was collected as input. Then, streptavidin beads were added into each binding reaction and incubated at room temperature for $1 \mathrm{~h}$. At last, the beads were washed 5 times with RIP buffer and incubated with $5 \times$ sample buffer at $95^{\circ} \mathrm{C}$ for $5 \mathrm{~min}$. The DNMT1 protein was analyzed using western blot analysis.

\section{Chromatin Immunoprecipitation (ChIP) Assay}

An EZ-Magna ChIP A kit (Merck Millipore, Billerica, MA, USA) was used for ChIP assay. Prostate cancer cells in logarithmic growth phase were fixed with $1 \%$ formaldehyde for $10 \mathrm{~min}$ for crosslinking, which was terminated with addition of $125 \mathrm{mM}$ glycine. The cells were centrifuged at 2,000 rpm for $5 \mathrm{~min}$ and resuspended in the cell lysis buffer. The cells $\left(2 \times 10^{6}\right.$ cells $/ 200 \mathrm{~mL}$ ), were then centrifuged at $5,000 \mathrm{rpm}$ for $5 \mathrm{~min}$ with proteinase inhibitor, resuspended with nuclear fraction buffer, and placed on an ice-bath for $10 \mathrm{~min}$. Next, cells were ultrasonicated to a length of 200-1,000 base pairs and centrifuged at $4 \mathrm{C}$, whereupon $100 \mu \mathrm{L}$ supernatant (containing DNA fragments) was added into $900 \mu \mathrm{L}$ ChIP dilution buffer, $20 \mathrm{~mL} 50^{\circ} \times$ PIC and $60 \mu \mathrm{L}$ Protein A Agarose/Salmon Sperm DNA and incubated at $4^{\circ} \mathrm{C}$ for $1 \mathrm{~h}$. The mixture was allowed to stand at $4^{\circ} \mathrm{C}$ for $10 \mathrm{~min}$ and centrifuged at $700 \mathrm{rpm}$ for $1 \mathrm{~min}$. The supernatant $(20 \mu \mathrm{L})$ was incubated overnight with rabbit antibodies against DNMT1 (ab19905, 1:100), DNMT3b (ab79822, 1:1,000), and DNMT3a (ab4897, 1:1,000) or IgG (NC, ab172730), all purchased from Abcam Inc. Then, a total of 60 $\mu \mathrm{L}$ Protein A Agarose/Salmon Sperm DNA was added for a 2$\mathrm{h}$ incubation at $4^{\circ} \mathrm{C}$. After $10 \mathrm{~min}$, the solution was centrifuged at $700 \mathrm{rpm}$ for $1 \mathrm{~min}$. The precipitate was successively washed with $1 \mathrm{~mL}$ portions of low salt buffer, high salt buffer, $\mathrm{LiCl}$ solution and Tris-ethylenediamine tetraacetic acid. Each tube was eluted two times with $250 \mathrm{~mL}$ ChIP wash buffer. Decrosslinking was performed by adding $20 \mathrm{~mL} 5 \mathrm{M} \mathrm{NaCl}$, and then DNA was then collected. The LARGE DNA promoter in the complex was quantified by fluorescence quantitative PCR. The primer sequences of LARGE promoter are listed in Table 4.

\section{EdU Proliferation Assay}

Synchronized cells in logarithmic growth phase were seeded into 24 -well plates at $1 \times 10^{5}$ cells/well. After being incubated with $50 \mu \mathrm{M}$ EdU medium for $2 \mathrm{~h}$, the cells in each well was reacted successively with $100 \mu \mathrm{L} 4 \%$ polyformaldehyde in PBS at room temperature for $30 \mathrm{~min}, 100 \mu \mathrm{L} 2 \mathrm{mg} / \mathrm{mL}$ glycine for $5 \mathrm{~min}, 200$ $\mu \mathrm{L}$ 0.5\% Triton X-100 in PBS for $10 \mathrm{~min}$, and stained with 200 $\mu \mathrm{L} 1 \times$ Apollo staining solution (C10812-1, Guangzhou RiboBio Co., Ltd., Guangzhou, Guangdong, China) for $30 \mathrm{~min}$ at room 
temperature. The cells in each well were washed three times with $200 \mu \mathrm{L}$ permeate solution and incubated with $200 \mu \mathrm{L} 1$ $\times$ Hoechst 33342 reaction solution (DA0014, Beijing Leagene Biotech Co., Ltd., Beijing, China) for $30 \mathrm{~min}$ in the dark. Under a fluorescence microscope, the EdU fluorescence signal was red and Hoechst 33342 fluorescence signal was blue. Therefore, cells with red fluorescence were proliferating cells and cells with blue fluorescence were non-proliferating cells.

\section{Transwell Migration and Invasion Assays}

Cell migration and invasion were assessed in vitro using a 24well Transwell insert system (Corning Glass Works, Corning, NY, USA). A total of $600 \mu \mathrm{L}$ RPMI 1640 medium containing $20 \%$ FBS was seeded into the basolateral Transwell chambers precoated with (for invasion) or without (for migration) Matrigel and incubated at $37^{\circ} \mathrm{C}$ for $1 \mathrm{~h}$. At $48 \mathrm{~h}$ after transduction, prostate cancer cells were resuspended in RPMI 1640 medium with $10 \%$ FBS. Next, $0.2 \mathrm{~mL}$ cell suspension $\left(3 \times 10^{5}\right.$ cells $)$ at the density of $1.5 \times 10^{6}$ cells $/ \mathrm{mL}$ was added to apical chamber and incubated at $37^{\circ} \mathrm{C}$ with $5 \% \mathrm{CO}_{2}$. After incubation for $24 \mathrm{~h}$, cells in the upper membrane were removed with cotton swabs, while those that migrated or invaded were fixed with $4 \%$ methanol and stained with $0.1 \%$ crystal violet. The number of stained cells was counted and photographed under an inverted microscope (Olympus IX73; Olympus Optical Co., Ltd., Tokyo, Japan). Five visual fields were randomly selected to analyze the differences among groups.

\section{Xenograft Tumor in SCID Mice}

The stably transduced LNCaP cells were established by infecting lentivirus harboring oe-NC or oe-DLX6-AS1. The stably transduced cells were cultured in vitro for 3-7 days and then dispersed into a single cell suspension $\left(1 \times 10^{6}\right.$ cells/50 $\left.\mathrm{\mu L}\right)$. For in situ injection, mice were anesthetized by 3\% pentobarbital sodium (No: P3761, Sigma-Aldrich Chemical Company) to expose the prostate through abdominal incision. Next, the LNCaP cell suspension was injected into the prostate of 54 nude mice. The day of injection was designated as day 0 . From the 7 th day after injection, 3 mice were euthanized and the size of tumors was measured with Vernier caliper every week at each time point (15 in each group). The volume (V) was calculated as: $\mathrm{V}=0.8$ $\times 2 / 3 \times D_{1}^{2} \times D_{2}$, where $D_{1}$ and $D_{2}$ indicate the maximum and minimum diameter, respectively. In the last week, orthotopic xenografted tumors were removed by surgical operation, and the weights of the tumors were recorded (12 in each group). Samples of ipsilateral lymph node metastasis on transplanted tumor pelvis were harvested, and RNA was extracted for RTqPCR. Mice were euthanized by intravenous injection of $3 \%$ sodium pentobarbital.

\section{Hematoxylin-Eosin (HE) Staining}

The specimens obtained from formaldehyde fixative were washed with distilled water for $1 \mathrm{~h}$ and then fully immersed in graded alcohol, embedded with paraffin and cut into $5 \mu \mathrm{m}$ thick serial sections. The sections were heated at $60^{\circ} \mathrm{C}$ for $1 \mathrm{~h}$, deparaffinized with xylene, stained with hematoxylin for 5-10 min, and differentiated with $1 \%$ hydrochloric acid solution. Then the slides were stained with eosin solution for 1-2 min, and finally dehydrated, cleared, and sealed with neutral gum. The morphological structure and cell infiltration of xenografted tumors and lymph nodes were observed under the ordinary optical microscopy, and the pathological morphology was described. ImageJ software was applied to collect images.

\section{Immunohistochemistry}

Tumor tissues and lymph node tissues collected from the mice were fixed with $4 \%$ paraformaldehyde solution, embedded in paraffin, and sliced into serial $4-\mu \mathrm{m}$ sections. The sections were then deparaffinized and the antigen was retrieved by the streptavidin-peroxidase method. Then the sections were sealed with normal goat serum blocking solution using the Histostain TMSP-9000 immunohistochemical staining kit (Zymed Laboratories Inc., South San Francisco, USA) and incubated with primary rabbit antibody to LARGE (ab228953, 1:200, Abcam Inc.) overnight at $4^{\circ} \mathrm{C}$. Subsequently, the sections were reacted with secondary mouse anti-rabbitantibody (ab9482, 1:5,000, Abcam Inc) at $37^{\circ} \mathrm{C}$ for $30 \mathrm{~min}$ and incubated with horseradish peroxidase-labeled streptomyces ovalbumin working solution. The sections were visualized with diaminobenzidine for 5-10 min and counter-stained with hematoxylin for $1 \mathrm{~min}$. Finally, the sections were photographed and scanned with an orthostatic optical microscope (Nikon Corporation, Tokyo, Japan). Five representative high-power visual fields were randomly selected for observation and calculation. The cells with obvious brown, and yellow particles in the cytoplasm were considered as positive cells.

\section{Immunofluorescence Assay}

The specimens of mice xenografted tumor tissues and lymph node tissues were fixed overnight with fresh $4 \%$ polyformaldehyde. The frozen specimens were cut into $4 \mu \mathrm{m}$ thick sections and placed at room temperature to melt the OCT. The tissues were penetrated and fixed with methanol (pre-cooled at $-20^{\circ} \mathrm{C}$ ) for $15 \mathrm{~min}$. After $60 \mathrm{~min}$ of sealing with $2 \%$ bovine serum albumin and $5 \%$ goat serum, the slides were stained with rabbit antibody to LARGE (ab228953, 1:200, Abcam Inc.) and Cy3-labeled secondary antibody (ab6939, Abcam Inc.) at room temperature for $60 \mathrm{~min}$ in the dark. Tissues were stained with 1 $\mathrm{mg} / \mathrm{mL}$ DAPI, sealed with fluorescent mounting medium, and stored under $4^{\circ} \mathrm{C}$ away from light for up to 1 week. Six visual fields were randomly selected in each group for quantitative analysis of LARGE positive cells by using Image J software. All images were obtained by using LEICA DC 500 camera on a microscope equipped with LEICA DMRA2 fluorescence optics (Leica Microsystems GmbH, Wetzlar, Germany).

\section{Statistical Analysis}

All data were analyzed by SPSS 21.0 software (IBM, Armonk, NY, USA), with $p<0.05$ as the level of statistically significance. Measurement data was expressed as mean \pm standard deviation. Paired data in compliance with normal distribution and 
homogeneity between two groups were compared using paired $t$-test and unpaired data using unpaired $t$-test. Comparisons among multiple groups were conducted by one-way analysis of variance (ANOVA), followed by Tukey's post-hoc test. Data at different time points were compared by repeated measures ANOVA with Bonferroni post-hoc test. Pearson method was applied to characterize the correlation between DLX6-AS1 and LARGE expression in prostate cancer tissues.

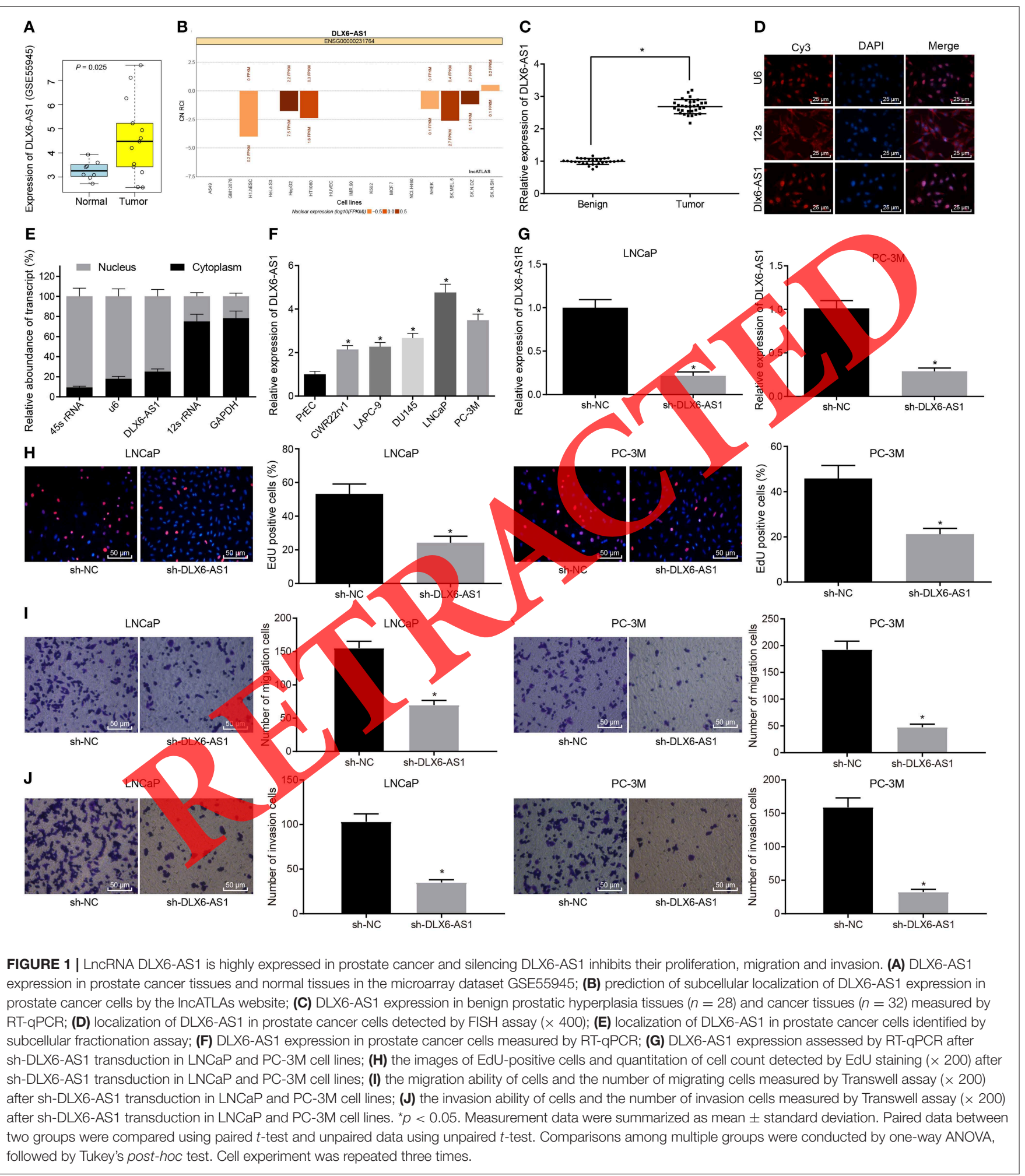




\section{RESULTS}

\section{DLX6-AS1 Expresses at a High Level in Prostate Cancer Tissues, Cells, and Promotes Cell Malignant Phenotypes}

First, we performed bioinformatics analysis to identify differentially expressed IncRNAs in prostate cancer, and screened out the highly expressed DLX6-AS1 in the dataset GSE55945 (Figure 1A). Moreover, the expression of DLX6-AS1 in the nucleus was predicted through the website of IncATLAS (http://ncatlas.crg.eu/; Figure 1B). Further, we found that DLX6-AS1 was highly expressed in prostate cancer tissue samples, relative to benign prostatic hyperplasia tissue samples (Figure 1C). Based on FISH and subcellular fractionation assays, DLX6-AS1 was mainly expressed in the nucleus of prostate cancer cells (Figures 1D,E). The expression of DLX6-AS1 in five prostate cancer cell lines (CWR22rv1, LAPC-9, DU145, LNCaP, and PC-3M) was measured by RT-qPCR. Two cell lines with the highest expression (LNCaP and PC-3M) were selected for subsequent experiments (Figure 1F).

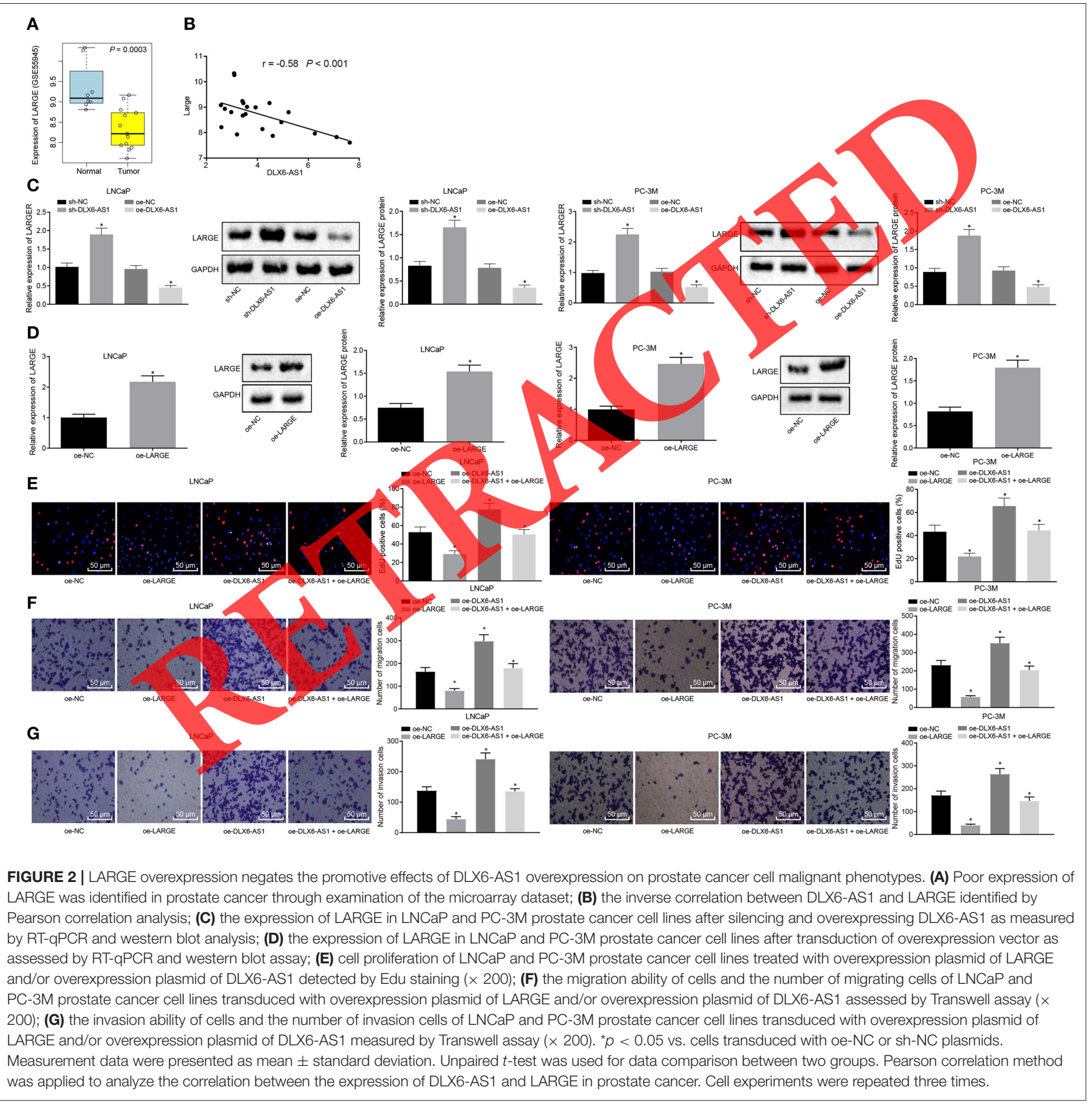


LNCaP and PC-3M cells were treated with sh-DLX6-AS1, and the silencing efficiency was determined using RT-qPCR, which demonstrated that the expression of DLX6-AS1 was reduced (Figure 1G). The proliferation ability of cells after silencing DLX6-AS1 was assessed by EdU assay, which revealed that the proliferation ability of LNCaP and PC-3M cells was decreased (Figure 1H). The Transwell assay showed that the migration and invasion abilities of LNCaP and PC-3M cells were diminished (Figures 1I,J).

These data suggested that DLX6-AS1 was highly expressed in prostate cancer tissues and cells, and mainly expressed in the nucleus, and DLX6-AS1 depletion could reduce the proliferation, migration, and invasion of prostate cancer cells.

\section{DLX6-AS1 Promotes Prostate Cancer Cell Malignant Phenotypes by Negatively Regulating LARGE}

To understand better the downstream mechanism of DLX6AS1, we searched the GSE55945 data set, which showed that LARGE was a poorly expressed gene in prostate cancer
(Figure 2A), and predicted that DLX6-AS1 would be negatively correlated with LARGE (Figure 2B). Moreover, silencing DLX6AS1 in LNCaP and PC-3M cell lines increased the expression of LARGE, while DLX6-AS1 overexpression decreased its expression (Figure 2C). Further, we constructed overexpression vectors of LARGE in LNCaP and PC-3M cell lines and assessed the transduction efficiency. The data suggested that the transduction of overexpression vector of LARGE increased the mRNA and protein expression of LARGE, indicating that the vectors were successfully delivered (Figure 2D).

The EdU assay further suggested that LNCaP and PC-3M cells overexpressing LARGE had restrained cell proliferation, but that overexpressing DLX6-AS1 accelerated proliferation. Besides, LARGE overexpression diminished the cell proliferation otherwise induced by DLX6-AS1 overexpression (Figure 2E). Transwell assay in LNCaP and PC-3M cells showed that LARGE overexpression suppressed migration and invasion abilities of the cells, while DLX6-AS1 overexpression increased migration and invasion abilities of the cells. Furthermore, LARGE overexpression reversed the stimulating role of DLX6AS1 in cell invasion and migration (Figures 2E,6).

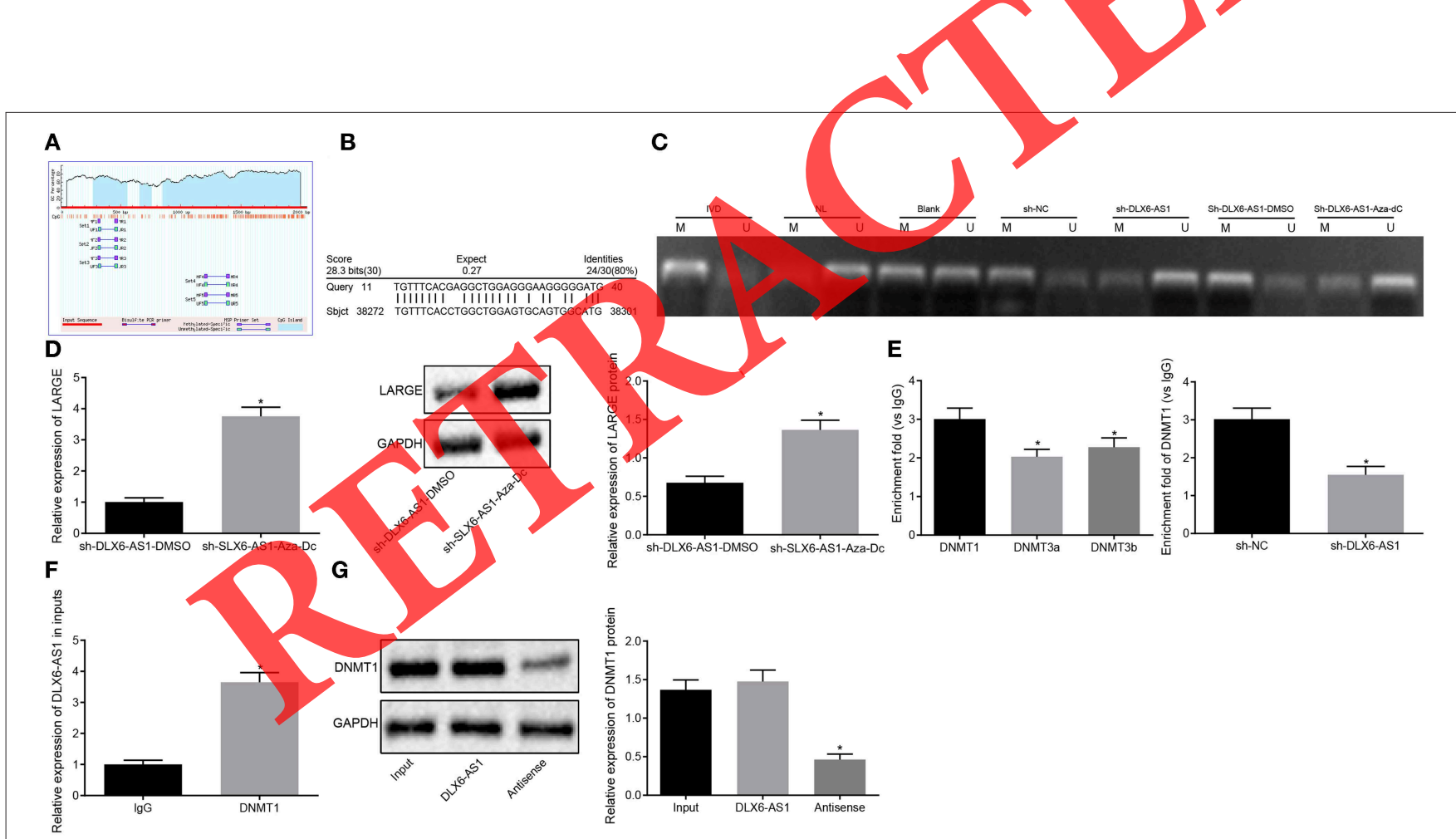

FIGURE 3 | DLX6-AS1 promotes methylation of LARGE promoter by recruiting DNMT1. (A) The condition of CpG islands in LARGE promoter region predicted by the MethPrimer website; (B) the binding of DLX6-AS1 and LARGE analyzed by Blast online comparative analysis; (C) MSP assessment of methylation status in LARGE promoter region under different treatments; IVD: in vitro methylated DNA, positive control; NL, normal lymphocytes, unmethylated positive control; Blank, blank control; U, unmethylation; M, methylation; sh-NC, cells transduced with shRNA negative control; sh-DLX6-AS1, cells transduced with shRNA targeting DLX6-AS1; sh-DLX6-AS1 + DMSO, cells that received shRNA targeting DLX6-AS1 and DMSO treatment; sh-DLX6-AS1 + Aza-dC, cells that received shRNA targeting DLX6-AS1 and methyltransferase inhibitor Aza-dC. (D) the expression of LARGE in cells after Aza-dC treatment measured by RT-qPCR and western blot assay; (E) the enrichment of DNMT1, DNMT3a, and DNMT3b in LARGE promoter region, and the enrichment of DNMT1 after sh-DLX6-AS1 transduction assessed by ChIP; (F) the enrichment of DLX6-AS1 pulled down by lgG and DNMT1 measured by RIP assay; (G) the binding of DLX6-AS1 to DNMT1 assessed by RNA pull-down assay. ${ }^{*} p$ $<0.05$. Measurement data were summarized as mean \pm standard deviation. Unpaired $t$-test was used for data comparison between two groups, and comparisons among multiple groups were analyzed by the one-way ANOVA with Tukey's post-hoc test. Cell experiment was repeated three times. 
The above results supported that the expression of LARGE was negatively correlated with the expression of DLX6-AS1 in prostate cancer cells. DLX6-AS1 induced cell proliferation, migration and invasion through downregulating LARGE.

\section{DLX6-AS1 Regulates LARGE Transcription by Recruiting DNMT1}

To explore further the interaction between DLX6-AS1 and LARGE, we found through the MethPrimer website (http://www.urogene.org/cgi-bin/methprimer/methprimer.cgi)
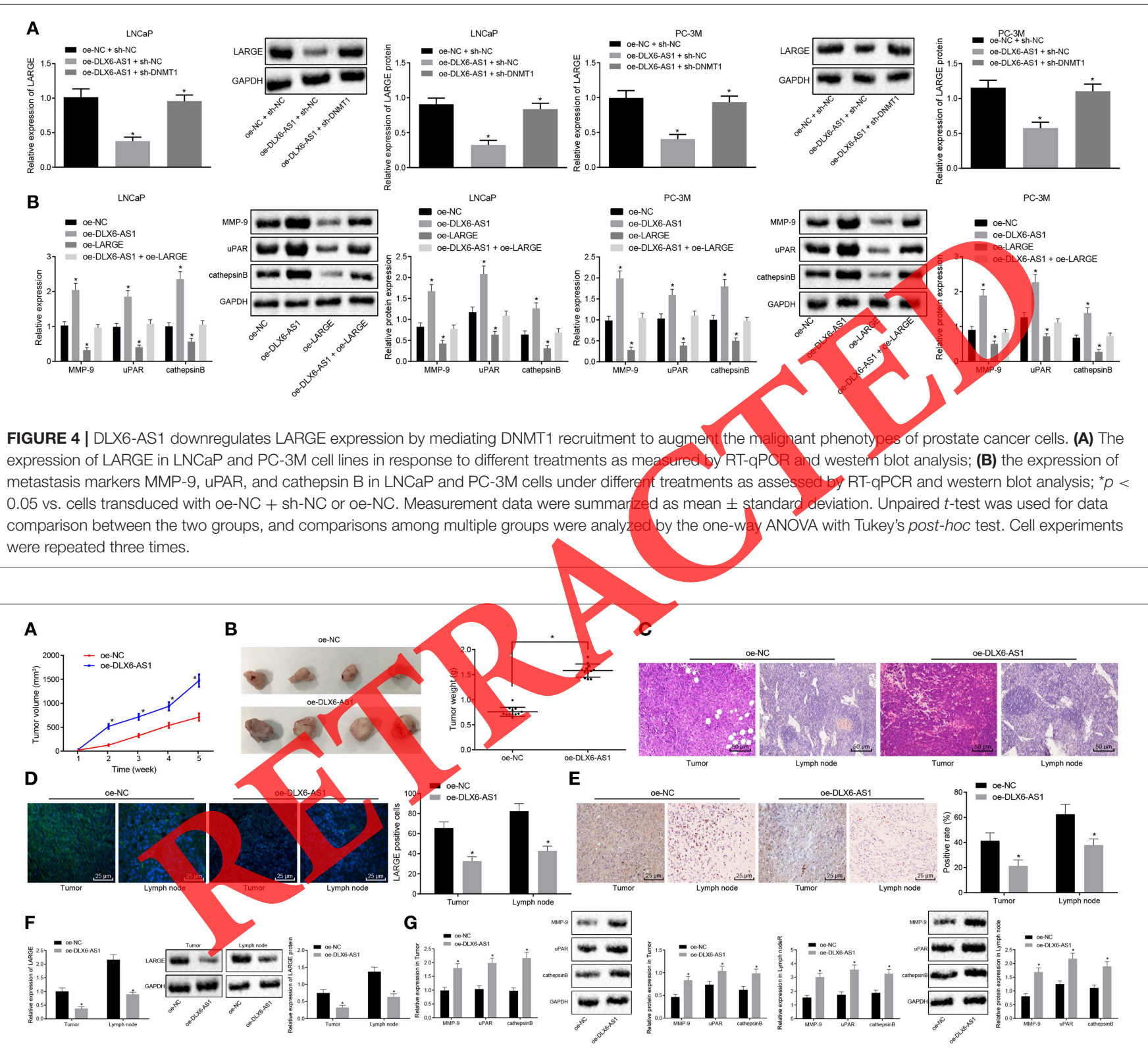

FIGURE 5 | DLX6-AS1 downregulates LARGE to augment lymph node metastasis and the progression of prostate cancer in vivo. (A) The volume of xenografted tumors from mice transduced with overexpression vector of DLX6-AS1 or oe-NC $(n=3)$; (B) the weights of xenografted tumors from mice transduced with overexpression vector of DLX6-AS1 $(n=12)$ or oe-NC $(n=12)$; (C) HE staining $(\times 200)$ for cell infiltration after the treatment of DLX6-AS1 overexpression in xenografted tumors and lymph nodes; (D) the number of positive cells of LARGE in xenografted tumors and lymph nodes after the treatment of overexpressing DLX6-AS1 counted by immunofluorescence assay ( $\times$ 400); (E) LARGE positive expression in in xenografted tumors and lymph nodes after the treatment of overexpression vector of DLX6-AS1 determined by immunohistochemistry ( $\times 400)$; (F) the expression of LARGE in xenografted tumors and lymph nodes from nude mice transduced with overexpression vector of DLX6-AS1 as measured by RT-qPCR and western blot assay; (G) the expression of MMP-9, uPAR, and cathepsin B in xenografted tumors and lymph nodes from nude mice after the treatment of overexpression vector of DLX6-AS1 assessed by RT-qPCR and western blot assay. ${ }^{*} p<$ 0.05 vs. the treatment of oe-NC. Measurement data were expressed as mean \pm standard deviation. Unpaired $t$-test was used for data comparison between the two groups. Data comparisons among multiple groups at different time points were analyzed by the repeated measures ANOVA with Bonferroni's post-hoc test. 
that the promoter region of LARGE was enriched in $\mathrm{CpG}$ islands (Figure 3A). The Basic Local Alignment Search Tool (Blast) revealed that DLX6-AS1 might bind to the LARGE promoter in the form of an RNA-DNA complex (Figure 3B). Therefore, we speculated that DLX6-AS1 may induce LARGE promoter hypermethylation, thereby inhibiting the expression of LARGE. To test the above hypothesis, the methylation status of LARGE promoter was examined by an MSP experiment, which showed that, compared with cells transduced with sh-NC, the methylation level of LARGE in cells treated with sh-DLX6-AS1 was appreciably decreased (Figure 3C). The methylation, mRNA and protein expression of LARGE were measured in cells treated with Aza-dC or DMSO in the presence of sh-DLX6-AS1. This analysis showed that the methylation level of cells treated with Aza-dC and sh-DLX6-AS1 was decreased (Figure 3C). However, the expression of LARGE in cells treated with sh-DLX6-AS1 and Aza-dC was increased (Figure 3D). The above data suggested that DLX6-AS1 downregulated the expression of LARGE by influencing promoter methylation of LARGE.

To study further the mechanism of the methylation of DLX6AS1 in regulating LARGE, we measured the enrichment of three methyltransferases (DNMT1, DNMT3a, and DNMT3b) in the LARGE promoter region by ChIP experiments. DNMT1 showed the highest degree of enrichment in the LARGE promoter region, and silencing DLX6-AS1 reduced that enrichment (Figure 3E). Additionally, DLX6-AS1 could be pulled down by DNMT1 antibody in the RIP assay (Figure 3F), and DNMT1 was observed in RNA obtained by RNA pull down (Figure 3G).
The results revealed that DLX6-AS1 restricted LARGE expression by recruiting DNMT1 to the LARGE promoter region.

\section{DLX6-AS1/DNMT1 Regulates LARGE in Prostate Cancer Cells and Promotes the Progression of Prostate Cancer}

To investigate the roles of DLX6-AS1/DNMT1/LARGE axis in LNCaP and PC-3M prostate cancer cells, we applied gainof-function and loss-of-function approaches. Relative to cells transduced with oe-NC and sh-NC, the cells treated with oeDLX6-AS1 and sh-NC had downregulated the mRNA and protein expression of LARGE, while the cells treated with oe-DLX6-AS1 and sh-DNMT1 had restored expression of LARGE (Figure 4A). Together, DLX6-AS1 affected the biological function of prostate cancer LNCaP and PC-3M cells by recruiting DNMT1 to diminish LARGE expression

To explore further the relationship between LARGE and lymph node metastasis in prostate cancer, we transduced the cells with oe-DLX6-AS1 and/or oe-LARGE, which showed that overexpression of DLX6-AS1 enhanced the expression of MMP-9, uPAR, and cathepsin B, while LARGE overexpression repressed their expression. The metastasis-promoting effect of DLX6-AS1 was counteracted by LARGE overexpression in prostate cancer LNCaP and PC-3M cells (Figure 4B).

The above data suggested that DLX6-AS1 downregulated LARGE expression by mediating DNMT1 methyltransferase in prostate cancer cells, leading to facilitated progression of in prostate cancer

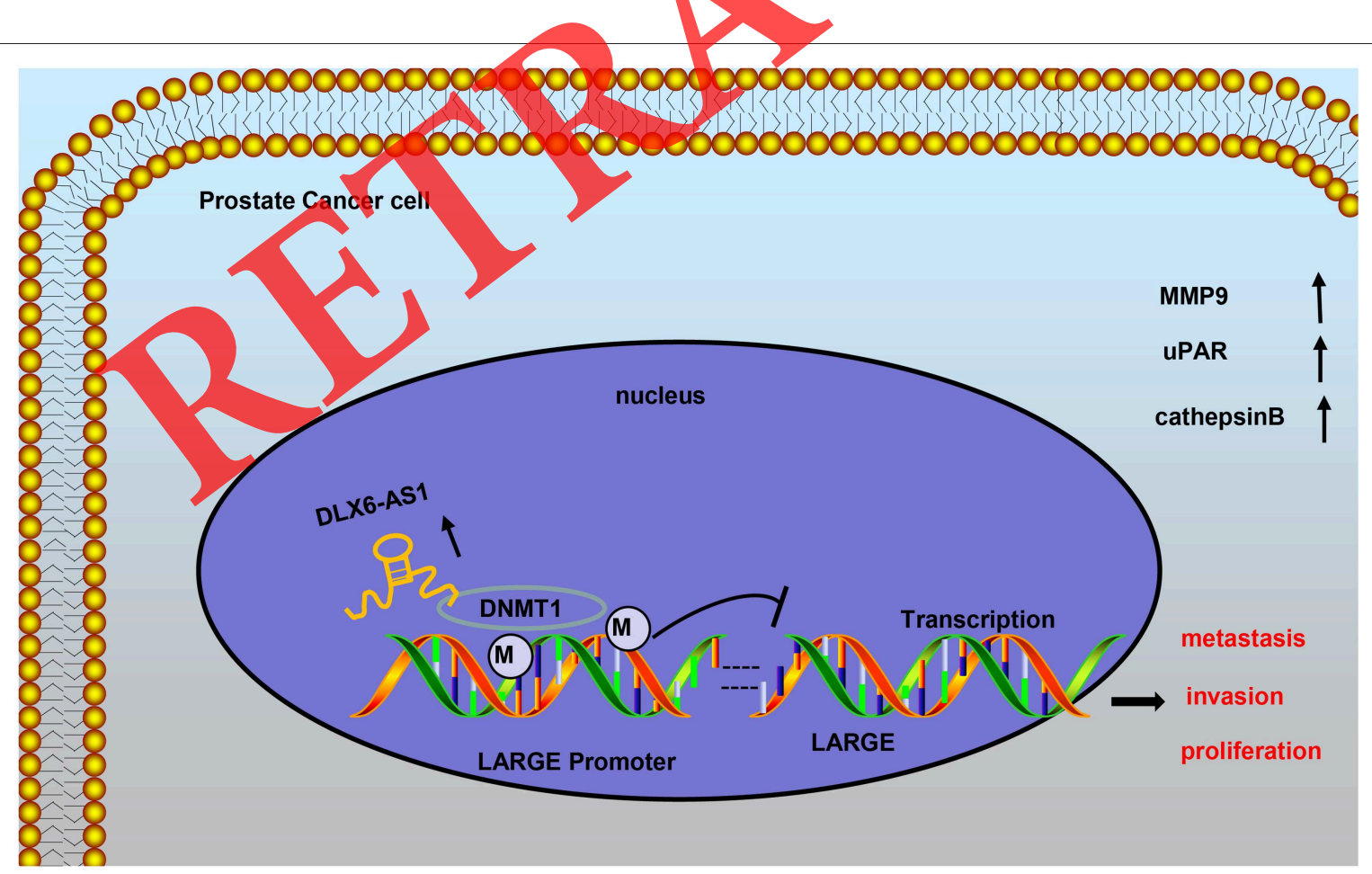

FIGURE 6 | Schematic representation and function of DLX6-AS1 in malignant behaviors of prostate cancer cells. DLX6-AS1 downregulates the expression of LARGE through the recruitment of DNMT1 to LARGE promoter and inducing its methylation, to accelerate proliferation, invasion, and metastasis of prostate cancer cells. 


\section{DLX6-AS1 Promotes the Progression of Prostate Cancer by Regulating LARGE in vivo}

On the basis of our in vitro results, we sought to verify the effects of DLX6-AS1 overexpression in vivo by developing a xenograft mouse model with prostate cancer. The tumors from nude mice treated with oe-DLX6-AS1 were larger than those with oe-NC (Figure 5A). The weight of tumors in mice after the treatment of oe-DLX6-AS1 was greater than that of the oe-NC group (Figure 5B), indicating that DLX6-AS1 accelerated the formation of tumors in vivo.

According to the functional characteristics of DLX6-AS1 in promoting migration of prostate cancer cells in vitro, we speculated that DLX6-AS1 could augment lymph node metastasis in vivo. HE staining confirmed the infiltration of cells in pelvic lymph nodes and tumor tissues was more severe in nude mice treated with oe-DLX6-AS1 vs. those with oe-NC (Figure 5C). Furthermore, immunofluorescence assay demonstrated that the LARGE expression in xenografted tumors and lymph nodes was lower in mice treated with oeDLX6-AS1 than in the oe-NC group (Figure 5D). LARGE expression in tumor tissues and lymph node samples was detected by immunohistochemistry, RT-qPCR and western blot, all of which revealed consistent results (Figures 5E,F). In addition, the expression of MMP-9, uPAR, and cathepsin $B$ was increased in both tumors and lymph node samples, the promotion of which was more pronounced in lymph nodes (Figure 5G). The above data revealed that DLX6-AS1 accelerated lymph node metastasis by inhibiting the expression of LARGE in vivo.

\section{DISCUSSION}

LncRNAs have already been proved to be abnormally expressed in prostate cancer tissues and are related to the malignant phenotypes of prostate cancer cells, including EMT, metastasis, and invasion $(1,14)$. Thus, one or more of the abnormally expressed lncRNAs may serve as promising therapeutic targets for lreating patients with prostate cancer. In the present study, the functional effects and potential underlying mechanism of DLX6-AS1 in prostate cancer in vitro and in vivo were delineated. We found clear evidence that DLX6-AS1 contributed to the progression of prostate cancer by accelerating malignant phenotypes of cells and lymph node metastasis through facilitating the DNMT1-mediated LARGE methylation (Figure 6).

In our study, DLX6-AS1 had a high expression high level in prostate cancer, and its silencing restricted cell proliferation, migration, and invasion of prostate cancer cells in vitro as well as lymph node metastasis in vivo. The involvement of lncRNAs in the tumorigenesis, metastasis, and prognosis or diagnosis of prostate cancer has been highlighted in a previous study (15). Consistent with present findings, the level of DLX6AS1 was upregulated in gastric cancer, where DLX6-AS1 also promotes cell proliferation, migration and EMT (16). The
DLX6-AS1 overexpression in hepatocellular carcinoma was also found to facilitate tumorigenesis and tumor growth (12). Most importantly, the elevated DLX6-AS1 expression was monitored to be closely correlated to advanced disease stage, positive lymph node metastasis as well as poor tumor differentiation of nonsmall cell lung cancer (17). Moreover, our in vivo experiments demonstrated that DLX6-AS1 enhanced the expression of MMP9, uPAR, and cathepsin B. Likewise, reduction of MMP-9, UPAR, and cathepsin B repressed invasion and migration ability in a previous study of PC 3 and DU145 prostate cancer cell lines (18).

Our findings provided evidence demonstrating that LARGE has low expression in prostate cancer, and that this expression correlates negatively with the expression of DLX6AS1. Furthermore, the LARGE overexpression appreciably suppresses cell proliferation, migration, and invasion in prostate cancer. A previous study has found that the expression of LARGE is associated with some diseases (19). However, there are few studies on the relationship between LARGE and prostate cancer. The complieated relationship between DNA methylation and histone modifications has become a hot topic of increasing concern in the field of cancer research. In an animal model, Lee et al. observed that knockdown of DNMT1 by pretreatment of 5-Aza in vitro remarkably enhances primary tumor growth and bone metastasis potential of prostate cancer cells (20). However, the associations of DNMT1 with other potential genes aberrantly expressed in prostate cancer remain unknown.

In this study, we hypothesized the DLX6-AS1 may induce hypermethylation of LARGE by recruiting DNMT1 to the promoter region of LARGE. According to ChIP assays, the methyltransferase DNMT1 had a higher degree of enrichment in the LARGE promoter region relative to DNMT3a and DNMT3b, and silencing DLX6-AS1 reduced the enrichment of DNMT1 in the LARGE promoter region. The results thus revealed that the DLX6-AS1 restricted LARGE expression by recruiting DNMT1 to augment methylation of LARGE promoter region, thus affecting the function of prostate cancer cells. Furthermore, LARGE overexpression eliminated the stimulating effect of DLX6-AS1 on gene expression in prostate cancer cells, thus providing additional evidence of a functionally significant reciprocal repression between DLX6-AS1 and LARGE.

Taken together, we provide evidence demonstrating that silencing of DLX6-AS1 played a regulatory role in reducing the prostate cancer cell proliferation, invasion, and lymph node metastasis by inhibiting the DNMT1-mediated LARGE methylation. This observation suggests a promising new therapeutic strategy for the treatment of prostate cancer, which might eventually lead to clinical trials targeting the DLX6AS1 pathway.

\section{DATA AVAILABILITY STATEMENT}

All the data obtained and/or analyzed during the current study are available from the corresponding authors on reasonable request. 


\section{ETHICS STATEMENT}

This study was conducted under the approval of the Ethics Committee of Linyi People's Hospital of Shandong Province. All participants or their guardians signed informed consent. This animal study was reviewed and approved by Linyi People's Hospital of Shandong Province and the experiments involving animals were performed in compliance with the recommendations in the Guide for the Care and Use of Laboratory Animals of the National Institutes of Health.

\section{REFERENCES}

1. Saini S. PSA and beyond: alternative prostate cancer biomarkers. Cell Oncol. (2016) 39:97-106. doi: 10.1007/s13402-016-0268-6

2. Jin S, Wang J, Shen Y, Gan H, Xu P, Wei Y, et al. Comparison of different lymph node staging schemes in prostate cancer patients with lymph node metastasis. Int Urol Nephrol. (2019) 52:87-95. doi: 10.1007/s11255-019-02294-z

3. Connor MJ, Shah TT, Horan G, Bevan CL, Winkler M, Ahmed HU. Cytoreductive treatment strategies for de novo metastatic prostate cancer. Nat Rev Clin Oncol. (2019) 17:168-82. doi: 10.1038/s41571-019-0284-3

4. Gu P, Chen X, Xie R, Xie W, Huang L, Dong W, et al. A novel AR translational regulator lncRNA LBCS inhibits castration resistance of prostate cancer. $\mathrm{Mol}$ Cancer. (2019) 18:109. doi: 10.1186/s12943-019-1037-8

5. Flippot R, Beinse G, Boileve A, Vibert J, Malouf GG. Long non-coding RNAs in genitourinary malignancies: a whole new world. Nat Rev Urol. (2019) 16:484-504. doi: 10.1038/s41585-019-0195-1

6. Zeng X, Hu Z, Ke X, Tang H, Wu B, Wei X, et al. Long noncoding RNA DLX6AS1 promotes renal cell carcinoma progression via miR-26a/PTEN axis. Cell Cycle. (2017) 16:2212-9. doi: 10.1080/15384101.2017.1361072

7. de Bernabe DB, Inamori K, Yoshida-Moriguchi T, Weydert CJ, Harper HA, Willer $\mathrm{T}$, et al. Loss of alpha-dystroglycan laminin binding in epitheliumderived cancers is caused by silencing of LARGE. J Biol Chem. (2009) 284:11279-84. doi: 10.1074/jbc.C900007200

8. Ning $X$, Shi Z, Liu $X$, Zhang $A$, Han and EZH2 mediated methylation silences the microRNA-200b/a/429 gene and promotes tumor progression. Cancer Lett. (2015) 359;198-205. doi: 10.1016/j.canlet.2015.01.005

9. Merry CR, Forrest ME, Sabers JN, Beard L, Gao XH, Hatzoglou M, et al. DNMT1-associated long non-coding RNAs, regulate global gene expression and DNA methylation in colon cancer. Hum Mol Genet. (2015) 24:6240-53. doi: $10.1093 / \mathrm{hmg} / \mathrm{dd} 343$

10. Liu CC, Lin JH, Hsu TW, Su K, Li AF, Hsu HS, et al. IL-6 enriched lung cancer stem-like cell population by inhibition of cell cycle regulators via DNMT1 upregulation. Int J Cancer. (2015) 136.547-59. doi: 10.1002/ijc.29033

11. Wu T, Qu L, He G, Tian L, Li L, Zhou H, et al. Regulation of laryngeal squamous cell cancer progression by the lncRNA H19/miR-148a-3p/DNMT1 axis. Oncotarget. (2016) 7:11553-66. doi: 10.18632/oncotarget.7270

12. Wu DM, Zheng ZH, Zhang YB, Fan SH, Zhang ZF, Wang YJ, et al. Downregulated lncRNA DLX6-AS1 inhibits tumorigenesis through STAT3 signaling pathway by suppressing CADM1 promoter methylation in liver cancer stem cells. J Exp Clin Cancer Res. (2019) 38:237. doi: 10.1186/s13046-019-1239-3

\section{AUTHOR CONTRIBUTIONS}

ZZ conceived and designed the study. ZZ and SL collected the data, analyzed the data, and wrote the manuscript. ZZ and FS assisted with the data analyses and participated in the writing of manuscript. All authors read and approved the final manuscript.

\section{ACKNOWLEDGMENTS}

We acknowledge and appreciate our colleagues for their valuable suggestions and technical assistance for this study.

13. Soares JC, Soares AC, Rodrigues VC, Melendez ME, Santos AC, Faria EF, et al. Detection of the prostate cancer biomarker PCA3 with electrochemical and impedance-based biosensors. ACS Appl Mater Interfaces. (2019) 11:46645-50. doi: 10.1021/acsami.9b19180

14. $\mathrm{Hu} \mathrm{R}, \mathrm{Lu} \mathrm{Z}$. Long noncoding RNA HCP5 promotes prostate cancer cell proliferation by acting as the sponge of miR4656 to modulate CEMIP expression. Oncol Rep. (2019) 43:328-36. doi: 10.3892/or.2019.7404

15. Xu T, Lin CM, Cheng SQ, Min ,, Li L, Meng XM, et al. Pathological bases and clinical impact of long noncoding RNAs in prostate cancer: a new budding star. Mol Cancer. (2018) 17:103 doi: 10.1186/\$12943-018-0852-7

16. Wu Q, Ma J, Meng W, Hui P. DLX6-AS1 promotes cell proliferation, migration and EMT of gastric cancer through FUS-regulated MAP4K1. Cancer Biol Ther. (2020) 21:17-25. doi: 10.1080/15384047.2019.16

17. Zhang X, Guo H, Bao Y, Yu H, Xie D, Wang X. Exosomal long non-coding RNA DLX6-AS1 as a potential diagnostic biomarker for non-small cell lung cancer. Oncol Lett, (2019) 18:5197-204. doi: 10.3892/ol.2019.10892

18. Nalla AK, Gorantla B, Gondi CS, Lakka SS, Rao JS. Targeting MMP9, UPAR, and cathepsin B inhibits invasion, migration and activates apoptosis in prostate cancer cells. Cancer Genet Ther. (2010) 17:599-613. doi: $10.1038 /$ cgt.2010.16

19. Hara Y, Kanagawa M, Kunz S, Yoshida-Moriguchi T, Satz JS, Kobayashi YM, et al. Like-acetylglucosaminyltransferase (LARGE)-dependent modification of dystroglycan at Thr-317/319 is required for laminin binding and arenavirus infection. Proc Natl Acad Sci USA. (2011) 108:17426-31. doi: $10.1073 /$ pnas. 1114836108

20. Lee E, Wang J, Yumoto K, Jung Y, Cackowski FC, Decker AM, et al. DNMT1 regulates epithelial-mesenchymal transition and cancer stem cells, which promotes prostate cancer metastasis. Neoplasia. (2016) 18:553-66. doi: 10.1016/j.neo.2016.07.007

Conflict of Interest: The authors declare that the research was conducted in the absence of any commercial or financial relationships that could be construed as a potential conflict of interest.

Copyright (C) 2020 Zhao, Liang and Sun. This is an open-access article distributed under the terms of the Creative Commons Attribution License (CC BY). The use, distribution or reproduction in other forums is permitted, provided the original author(s) and the copyright owner(s) are credited and that the original publication in this journal is cited, in accordance with accepted academic practice. No use, distribution or reproduction is permitted which does not comply with these terms. 